\title{
Dynamical Behaviors of a Stochastic SIQR Epidemic Model with Quarantine-Adjusted Incidence
}

\author{
Zhongwei Cao $\mathbb{D}^{1}$ and Shengjuan Zhou $\mathbb{D}^{2}$ \\ ${ }^{1}$ Department of Applied Mathematics, Jilin University of Finance and Economics, Changchun 130117, China \\ ${ }^{2}$ College of Science, China University of Petroleum (East China), Qindao 266580, China
}

Correspondence should be addressed to Shengjuan Zhou; alinnazhou@hotmail.com

Received 28 September 2017; Revised 3 January 2018; Accepted 4 February 2018; Published 8 March 2018

Academic Editor: Josef Diblik

Copyright (c) 2018 Zhongwei Cao and Shengjuan Zhou. This is an open access article distributed under the Creative Commons Attribution License, which permits unrestricted use, distribution, and reproduction in any medium, provided the original work is properly cited.

\begin{abstract}
We study the dynamics of a stochastic SIQR epidemic disease with quarantine-adjusted incidence in this article. In order to find the sufficient conditions for the ergodicity and extermination of the model, we construct suitable stochastic Lyapunov functions and find the results of the stochastic SIQR epidemic model. From the results, we find that when the white noise is relatively large, the infectious diseases will become extinct; this also shows that the intervention of white noise will play an important part in controlling the spread of the disease.
\end{abstract}

\section{Introduction}

Recently, owing to the negative impact of infectious diseases on population growth, understanding the dynamic behavior of these diseases and predicting what will happened have become an important research topic (see e.g., [1-8]). Therefore, the establishment of mathematical models has become an important method to study the properties of infectious diseases. For more contagious diseases such as smallpox, measles, plague, mumps, and Ebola, the most direct and effective methods of interference are to isolate those who have already been infected, in order to decrease transmissions to susceptible individuals. From then on, one of the famous disease models, SIQR (see [9]), has been established, which can be described as follows:

$$
\begin{aligned}
& \dot{S}(t)=\Lambda-\frac{\beta S I}{S+I+R}-\mathrm{d} S, \\
& \dot{I}(t)=\left[\frac{\beta S}{S+I+R}-\left(\gamma+\delta+\mathrm{d}+\alpha_{1}\right)\right] I, \\
& \dot{Q}(t)=\delta I-\left(\mu+\mathrm{d}+\alpha_{2}\right) Q, \\
& \dot{R}(t)=\gamma I+\mu \mathrm{Q}-\mathrm{d} R .
\end{aligned}
$$

In this model, one assumed that the infection is given a permanent immunization after recovery. $S$ is the susceptible individual. When these people are infected with the disease, some enter I compartment, which will be infected; other people can quickly and completely recover and access $R$ compartment. In addition, when the susceptible individual enters $I$ compartment, it may be quarantined directly to enter $Q$ compartment. Before they recover, they all will go into $R$ compartment. Here, the total population of the model varies, because vulnerable parts of the population can be received through birth or immigrants and people will die of natural and disease deaths. Besides, in this model, the incidence given by $\beta S I /(S+I+R)$ is the quarantine-adjusted incidence. The total contacts of a susceptible person using this form of incidence are $\beta S I /(N-Q)=\beta(N-Q) /(N-Q)=\beta$ [9], so that, during quarantine process, the total number of contacts per day remains at $\beta$. From the model, the parameters can be summarized in the following list:

$\Lambda$ is the influx of people into the susceptible person's compartment.

$\mathrm{d}$ is the natural death rate of compartments $S, I, Q$, and $R$. 
$\beta$ is transmission coefficient from compartment $S$ to compartment $I$.

$\gamma$ is the recovery rate of infectious individuals.

$\delta$ is the isolation rate from $I$ to $Q$.

$\mu$ is the recovery rate of isolated individuals.

$\alpha_{1}$ is the disease-caused death rate of infectious individuals.

$\alpha_{2}$ is the disease-caused death rate of isolated individuals.

Assume that all parameters are nonnegative parameters. In particular, $\Lambda$ and $d$ are positive constants.

In model (1), the quarantine reproduction number is $R_{0}=\beta /\left(\gamma+\delta+\mathrm{d}+\alpha_{1}\right)$ [9], which determines whether the disease occurs. If $R_{0} \leq 1$, system (1) has a unique diseasefree equilibrium $P_{0}=\left(S_{0}, 0,0,0\right)=(\Lambda / \mathrm{d}, 0,0,0)$ and $P_{0}$ is globally asymptotically stable in invariant set $\mathscr{D}$, where $\mathscr{D}=\left\{(S, I, Q, R) \in \mathbb{R}_{+}^{4} \mid S+I+Q+R \leq \Lambda / d\right\}$. This reveals that the disease will die out and all people are susceptible to it. If $R_{0}>1$ and $\alpha_{1}=\alpha_{2}=0$, then $P_{0}$ is locally asymptotically stable in the region $\mathscr{D}$ and system (1) has only a positive endemic equilibrium $P^{*}=\left(S^{*}, I^{*}, Q^{*}, R^{*}\right)$ which we can find in [9], where

$$
\begin{aligned}
& S^{*} \\
& \quad=\frac{(\Lambda / \mathrm{d})\left[(\gamma+\mathrm{d})\left(\mu+\mathrm{d}+\alpha_{2}\right)+\delta \mu\right]}{\left(\mu+\mathrm{d}+\alpha_{2}\right)\left[R_{0}\left(\gamma+\delta+\mathrm{d}+\alpha_{1}\right)-\delta-\alpha_{1}\right]+\mu \delta}, \\
& I^{*}=\frac{\Lambda\left(R_{0}-1\right)\left(\mu+\mathrm{d}+\alpha_{2}\right)}{\left(\mu+\mathrm{d}+\alpha_{2}\right)\left[R_{0}\left(\gamma+\delta+\mathrm{d}+\alpha_{1}\right)-\delta-\alpha_{1}\right]+\mu \delta}, \\
& Q^{*} \\
& \quad=\frac{\delta \Lambda\left(R_{0}-1\right)}{\left(\mu+\mathrm{d}+\alpha_{2}\right)\left[R_{0}\left(\gamma+\delta+\mathrm{d}+\alpha_{1}\right)-\delta-\alpha_{1}\right]+\mu \delta},
\end{aligned}
$$

$R^{*}$

$$
=\frac{(\Lambda / \mathrm{d})\left(R_{0}-1\right)\left[\gamma\left(\mu+\mathrm{d}+\alpha_{2}\right)+\delta \mu\right]}{\left(\mu+\mathrm{d}+\alpha_{2}\right)\left[R_{0}\left(\gamma+\delta+\mathrm{d}+\alpha_{1}\right)-\delta-\alpha_{1}\right]+\mu \delta} .
$$

In addition, for some parameter values the Hopf bifurcation may occur.

In real life, disease systems are often affected by white noise (see [10-17]). So it is important to include the effect of stochastic perturbation in estimation of parameters. In many cases, stochastic systems can better describe the spread of infectious diseases (see [16-18]). For instance, the stochastic model can account for the stochastic infectious contact during latent and infectious period [19]. Compared with the deterministic system, this is more practical (see [10, 20-25]). Reference [10] cleared that the stochastic systems are more adapted to the problem of extinction of the disease. Paper [21] showed that the unique equilibrium in a deterministic model may disappear in corresponding stochastic system due to stochastic fluctuations [3].

There are so many methods to introduce stochastic perturbations in this system. From biological perspective, random effects can be expressed in Itô or Stratonovich stochastic integrals [26]. From mathematical perspective, random effects are manifested directly in input parameters which are assumed to have specific probability distributions such as uniform, beta, exponential distribution, and gamma [27]. In this paper, we used the stochastic SIQR epidemic model introduced by the approach of Hethcote et al. [9]; following this approach, we can establish a SDE SIQR epidemic model with quarantine-adjusted incidence.

Think the effects of random fluctuations out, we assume that the fluctuations in the environment are represented as a parameter $\beta$ change to random variable $\beta+\sigma_{1} \dot{B}_{1}(t)$ and we assume that random fluctuations are linear perturbations corresponding to the rate of change for each population. Then corresponding to model (1), we can establish the stochastic model:

$$
\begin{aligned}
d S= & {\left[\Lambda-\frac{\beta S I}{S+I+R}-\mathrm{d} S\right] d t-\sigma_{1} \frac{S I}{S+I+R} d B_{1}(t) } \\
& +\sigma_{2} S d B_{2}(t), \\
d I= & {\left[\frac{\beta S I}{S+I+R}-\left(\gamma+\delta+\mathrm{d}+\alpha_{1}\right) I\right] d t } \\
& +\sigma_{1} \frac{S I}{S+I+R} d B_{1}(t)+\sigma_{3} I d B_{3}(t), \\
d Q= & {\left[\delta I-\left(\mu+\mathrm{d}+\alpha_{2}\right) Q\right] d t+\sigma_{4} Q d B_{4}(t), } \\
d R= & (\gamma I+\mu Q-\mathrm{d} R) d t+\sigma_{5} R d B_{5}(t),
\end{aligned}
$$

where $B_{i}(t)$ are independent standard Brownian motions and $\sigma_{i}^{2}>0(i=1,2,3,4,5)$ represent the intensities of the white noise. The other coefficients are the same as system (1).

In this part, we will give some theoretical knowledge about stochastic differential equations. First of all, let $B(t)$ be l-dimensional standard Brownian motion which is defined on the complete probability space $\left(\Omega, \mathscr{F},\{\mathscr{F}\}_{t \geq 0}, \mathbb{P}\right)$ adapted to the filtration $\{\mathscr{F}\}_{t \geq 0}$ and let $X(t)$ be $l$-dimensional Itô's process on $t \geq 0$ with the stochastic differential equation [13]

$$
\begin{aligned}
& d X(t)=f(X(t), t) d t+g(X(t), t) d B(t), \\
& X\left(t_{0}\right)=X_{0} \in \mathbb{R}^{l} .
\end{aligned}
$$

Let $V \in C^{2,1}\left(\mathbb{R}^{l} \times\left[t_{0}, \infty\right] ; \mathbb{R}^{+}\right)$; then $V(X, t)$ is again Itô's process with the stochastic differential equation [13] which can be defined as follows:

$$
\begin{aligned}
& d V(X, t)=\left\{V_{t}(X, t)+V_{X}(X, t) f(X, t)\right. \\
& \left.+\frac{1}{2} \operatorname{trace}\left[g^{T}(X, t) V_{X X}(X, t) g(X, t)\right]\right\} d t \\
& +V_{X}(X, t) g(X, t) d B(t),
\end{aligned}
$$

where $V_{t}=\partial V / \partial t, V_{X}=\left(\partial V / \partial x_{1}, \ldots, \partial V / \partial x_{l}\right), V_{X X}=$ $\left(\partial^{2} V / \partial X_{i} \partial X_{j}\right)_{l \times l}$.

Lemma 1. The Markov process $X(t)$ has a unique ergodic stationary distribution $\pi(\cdot)$ if there exists a bounded domain $\mathscr{D} \subset \mathbb{E}_{l}$ with regular boundary $\Gamma$ and if 
$\left(A_{1}\right)$ there is a positive number $M$ such that $\sum_{i, j=1}^{l} a_{i j}(x) \xi_{i} \xi_{j} \geq M|\xi|^{2}, x \in D, \xi \in \mathbb{R}^{l}$,

$\left(A_{2}\right)$ there exists a nonnegative $C^{2}$-function $V$ that $L V$ is nonpositive for any [25] $\mathbb{E}_{l} \backslash \mathscr{D}$.

\section{Existence and Uniqueness of Positive Solution}

In this section, we will study the existence and uniqueness of positive solutions in system (3), which is also the premise of studying the long-term behavior of the model.

Theorem 2. For any initial value $X(0)=(S(0), I(0), Q(0)$, $R(0)) \in \mathbb{R}_{+}^{4}$, there is a unique positive solution $X(t)=$ $(S(t), I(t), Q(t), R(t)) \in \mathbb{R}_{+}^{4}$ of system (3) for $t \geq 0$ and the solution will maintain in $\mathbb{R}_{+}^{4}$ with probability 1 .

Proof. Since the coefficients of system (3) satisfy the local Lipschitz condition [3], then for any initial value $\left(S_{0}, I_{0}, Q_{0}, R_{0}\right) \epsilon$ $\mathbb{R}_{+}^{4}$, there is a unique local solution $(S(t), I(t), Q(t), R(t))$ on $\left[0, \tau_{e}\right)$, where $\tau_{e}$ is the explosion time [13]. To find that this solution is global, we only need to prove that $\tau_{e}=\infty$ a.s. Since the following argument is similar to that of [17], here we simply have to prove the difference with it. Let $n_{0}>0$ which make every component of $X(0)$ sufficiently large in interval $\left[1 / n_{0}, n_{0}\right]$. For every integer $n>n_{0}$, define the stopping time

$$
\begin{aligned}
\tau_{n} & =\inf \left\{t \in\left[0, \tau_{e}\right): \min \{S(t), I(t), Q(t), R(t)\}\right. \\
& \left.\leq \frac{1}{n} \text { or } \max \{S(t), I(t), Q(t), R(t)\} \geq n\right\},
\end{aligned}
$$

where we set inf $\varnothing=\infty$ throughout this article. Through the definition, we can get that $\tau_{n}$ increases with $n \rightarrow \infty$. Let $\tau_{\infty}=$ $\lim \sup _{n \rightarrow \infty} \tau_{n}$; then $\tau_{\infty} \leq \tau_{e}$ a.s. Next, we have to prove that $\tau_{\infty}=\infty$ a.s. If the assertion is against this, then there will be a pair of parameters $T>0$ and $\epsilon \in(0,1)$ so that $\mathbb{P}\left\{\tau_{\infty} \leq T\right\}>\epsilon$. Therefore, here exists an integer $n_{1}>n_{0}$ so that

$$
\mathbb{P}\left\{\tau_{n} \leq T\right\} \geq \epsilon, \quad n \geq n_{1}
$$

Define a $C^{2}$-function $V: R_{+}^{4} \rightarrow R_{+}$as follows:

$$
\begin{aligned}
V(S, I, Q, R)= & (S-1-\ln S)+(I-1-\ln I) \\
& +(Q-1-\ln Q)+(R-1-\ln R) .
\end{aligned}
$$

Using Itô's formula, one yields that

$$
\begin{aligned}
& d V(S, I, Q, R) \\
& =L V(S, I, Q, R) d t+\sigma_{1} \frac{I-S}{S+I+R} d B_{1}(t) \\
& \quad+\sigma_{2}(S-1) d B_{2}(t)+\sigma_{3}(I-1) d B_{3}(t) \\
& \quad+\sigma_{4}(Q-1) d B_{4}(t)+\sigma_{5}(Q-1) d B_{5}(t),
\end{aligned}
$$

where, $L V$

$$
\begin{aligned}
= & \left(1-\frac{1}{S}\right)\left(\Lambda-\frac{\beta S I}{S+I+R}-\mathrm{d} S\right) \\
& +\left(1-\frac{1}{I}\right)\left[\frac{\beta S I}{S+I+R}-\left(\gamma+\delta+\mathrm{d}+\alpha_{1}\right) I\right] \\
& +\left(1-\frac{1}{\mathrm{Q}}\right) \times\left[\delta I-\left(\mu+\mathrm{d}+\alpha_{2}\right) \mathrm{Q}\right] \\
& +\left(1-\frac{1}{R}\right)[\gamma I+\mu \mathrm{Q}-\mathrm{d}] \\
& +\frac{1}{2}\left[\left(I^{2}+S^{2}\right)\left(\frac{\sigma_{1}}{S+I+R}\right)^{2}+\sigma_{2}^{2}+\sigma_{3}^{2}+\sigma_{4}^{2}+\sigma_{5}^{2}\right] \\
= & +4 \mathrm{~d}-\mathrm{d}(S+I+Q+R)-\alpha_{1} I_{1}+\left(\gamma+\delta+\alpha_{1}\right) \\
& -\alpha_{2} \mathrm{Q}+\left(\mu+\mathrm{d}+\alpha_{2}\right)+\frac{\beta I}{S+I+R}-\frac{\Lambda}{S}-\frac{\gamma^{2}}{R} \\
& -\frac{\mu Q}{R} \\
& +\frac{1}{2}\left[\left(I^{2}+S^{2}\right)\left(\frac{\sigma_{1}}{S+I+R}\right)^{2}+\sigma_{2}^{2}+\sigma_{3}^{2}+\sigma_{4}^{2}+\sigma_{5}^{2}\right] \\
< & +4 \mathrm{~d}+\left(\gamma+\delta+\mu+\beta+\alpha_{1}+\alpha_{2}\right) \\
& \left.+\sigma_{2}^{2}+\sigma_{3}^{2}+\sigma_{4}^{2}+\sigma_{5}^{2}\right):=M .
\end{aligned}
$$

$M$ is a positive constant and other parts of proof of Theorem 3, which we can get from Mao [13], here are omitted. Therefore, we have completed the proof.

\section{Ergodic Properties}

In this section, we define

$$
\begin{aligned}
& R_{0}^{s} \\
& =\frac{\mathrm{d} \beta}{\left(\mathrm{d}+\sigma_{1}^{2} / 2+\sigma_{2}^{2} / 2\right)\left(\gamma+\delta+\mathrm{d}+\alpha_{1}+\sigma_{1}^{2} / 2+\sigma_{3}^{2} / 2\right)} .
\end{aligned}
$$

Theorem 3. Assume that $R_{0}^{s}>1$; there exists a stationary distribution $\pi(\cdot)$ and the ergodicity holds for any initial value $(S(0), I(0), Q(0), R(0)) \in \mathbb{R}_{+}^{4}$ in system (3).

Proof. Let

$$
\begin{aligned}
D & =\left\{\varepsilon_{1} \leq S, \varepsilon_{1}^{2} \leq I, \varepsilon_{1}^{3} \leq Q, \varepsilon_{1}^{3} \leq R, S+I+Q+R\right. \\
& \left.\leq \frac{1}{\varepsilon_{1}}\right\},
\end{aligned}
$$

where $\varepsilon_{1}>0$ is a sufficiently small constant. In the set $\mathbb{R}_{+}^{4} \backslash D$, one can find that the following conditions hold: 


$$
\begin{gathered}
c_{1} M \varepsilon_{1} \beta<1 \\
-\frac{\Lambda}{\varepsilon_{1}}+K \leq-1, \\
-\frac{\delta}{\varepsilon_{1}}+K \leq-1, \\
-\frac{\gamma}{\varepsilon_{1}}+K \leq-1, \\
-\frac{d}{\varepsilon_{1}}+K \leq-1,
\end{gathered}
$$

where $M>0$ satisfying

$$
\begin{aligned}
& -M \lambda+\Lambda+3 \mathrm{~d}+\mu+\beta+\alpha_{2}+\frac{\sigma_{1}^{2}+\sigma_{2}^{2}+\sigma_{4}^{2}+\sigma_{5}^{2}}{2} \\
& \quad \leq-2
\end{aligned}
$$

here $\lambda=3 \Lambda\left[\left(R_{0}^{s}\right)^{1 / 3}-1\right]>0$. In order to be more intuitive, we divide $\mathbb{R}_{+}^{4} \backslash D$ into the following five regions:

$$
\begin{aligned}
& D_{1}=\left\{(S, I, Q, R) \in \mathbb{R}_{+}^{4}, 0<S<\varepsilon_{1}\right\}, \\
& D_{2}=\left\{(S, I, Q, R) \in \mathbb{R}_{+}^{4}, 0<I<\varepsilon_{1}^{2}, S \geq \varepsilon_{1}\right\}, \\
& D_{3}=\left\{(S, I, Q, R) \in \mathbb{R}_{+}^{4}, 0<Q<\varepsilon_{1}^{3}, I \geq \varepsilon_{1}^{2}\right\}, \\
& D_{4}=\left\{(S, I, Q, R) \in \mathbb{R}_{+}^{4}, 0<R<\varepsilon_{1}^{3}, I \geq \varepsilon_{1}^{2}\right\}, \\
& D_{5}=\left\{(S, I, Q, R) \in \mathbb{R}_{+}^{4}, S+I+Q+R>\frac{1}{\varepsilon_{1}}\right\} .
\end{aligned}
$$

The diffusion matrix of (3) is given by

$$
A=\left(\begin{array}{cccc}
\left(\frac{\sigma_{1} S I}{S+I+R}\right)^{2}+\sigma_{2}^{2} S^{2} & -\left(\frac{\sigma_{1} S I}{S+I+R}\right)^{2} & 0 & 0 \\
-\left(\frac{\sigma_{1} S I}{S+I+R}\right)^{2} & \left(\frac{\sigma_{1} S I}{S+I+R}\right)^{2}+\sigma_{3}^{2} I^{2} & 0 & 0 \\
0 & 0 & \sigma_{4}^{2} Q^{2} & 0 \\
0 & 0 & 0 & \sigma_{5}^{2} R^{2}
\end{array}\right)
$$

Choosing $M=\min _{(S, I, Q, R) \in \overline{D_{\sigma}} \subset \mathbb{R}_{+}^{4}}\left\{\sigma_{2}^{2} S^{2}, \sigma_{3}^{2} I^{2}, \sigma_{4}^{2} Q^{2}, \sigma_{5}^{2} R^{2}\right\}$, we can get

$$
\begin{aligned}
\sum_{i, j=1}^{5} a_{i j}(S, I, Q, R) \xi_{i} \xi_{j}= & \left(\frac{\sigma_{1} S I}{S+I+R}\right)^{2}\left(\xi_{1}-\xi_{2}\right)^{2} \\
& +\sigma_{2}^{2} S^{2} \xi_{1}^{2}+\sigma_{3}^{2} I^{2} \xi_{2}^{2}+\sigma_{4}^{2} Q^{2} \xi_{3}^{2} \\
& +\sigma_{5}^{2} R^{2} \xi_{4}^{2} \\
\geq & \sigma_{2}^{2} S^{2} \xi_{1}^{2}+\sigma_{3}^{2} I^{2} \xi_{2}^{2}+\sigma_{4}^{2} Q^{2} \xi_{3}^{2} \\
& +\sigma_{5}^{2} R^{2} \xi_{4}^{2} \geq M\|\xi\|^{2},
\end{aligned}
$$$$
(S, I, Q, R) \in \overline{D_{\sigma}}, \xi=\left(\xi_{1}, \xi_{2}, \xi_{3}, \xi_{4}\right) \in \mathbb{R}_{+}^{4} .
$$

Thus condition $\left(A_{1}\right)$ in Lemma 1 holds.

Now, we can define

$$
V_{1}=S+I+R+Q-c_{1} \ln S-c_{2} \ln I,
$$

where $c_{1}, c_{2}$ are the positive constants to be determined. Using Itô's formula, one gets

$$
\begin{aligned}
& L V_{1}=\Lambda-\mathrm{d}(S+I+R)-\delta I-\alpha_{1} I+\mu \mathrm{Q}+\delta I-(\mu \\
& \left.+\mathrm{d}+\alpha_{2}\right) \mathrm{Q}+c_{1}\left[-\frac{\Lambda}{S}+\frac{\beta I}{S+I+R}+\mathrm{d}\right. \\
& \left.\quad+\frac{\sigma_{1}^{2} I^{2}}{2(S+I+Q)^{2}}+\frac{\sigma_{2}^{2}}{2}\right]+c_{2}\left[\left(\gamma+\delta+\mathrm{d}+\alpha_{1}\right)\right.
\end{aligned}
$$

$$
\begin{aligned}
& \left.-\frac{\beta S}{S+I+R}+\frac{\sigma_{1}^{2} S^{2}}{2(S+I+Q)^{2}}+\frac{\sigma_{3}^{2}}{2}\right] \\
& =-\left[\mathrm{d}(S+I+R)+\frac{c_{1} \Lambda}{S}+\frac{c_{2} \beta S}{S+I+R}\right]+\Lambda-\alpha_{1} I \\
& -\mathrm{d} Q-\alpha_{2} Q+\frac{c_{1} \beta I}{S+I+R}+c_{1}\left[\mathrm{~d}+\frac{\sigma_{1}^{2} I^{2}}{2(S+I+Q)^{2}}\right. \\
& \left.+\frac{\sigma_{2}^{2}}{2}\right]+c_{2}\left[\left(\gamma+\delta+\mathrm{d}+\alpha_{1}\right)+\frac{\sigma_{1}^{2} S^{2}}{2(S+I+Q)^{2}}\right. \\
& \left.+\frac{\sigma_{3}^{2}}{2}\right]<-3\left[\mathrm{~d} \Lambda \beta c_{1} c_{2}\right]^{1 / 3}+\Lambda+\frac{c_{1} \beta I}{S+I+R}+c_{1}[\mathrm{~d} \\
& \left.+\frac{\sigma_{1}^{2}}{2}+\frac{\sigma_{2}^{2}}{2}\right]+c_{2}\left[\left(\gamma+\delta+\mathrm{d}+\alpha_{1}\right)+\frac{\sigma_{1}^{2}}{2}+\frac{\sigma_{3}^{2}}{2}\right] .
\end{aligned}
$$

Let

$$
\begin{aligned}
c_{1}\left[\mathrm{~d}+\frac{\sigma_{1}^{2}}{2}+\frac{\sigma_{2}^{2}}{2}\right] & =c_{2}\left[\left(\gamma+\delta+\mathrm{d}+\alpha_{1}\right)+\frac{\sigma_{1}^{2}}{2}+\frac{\sigma_{3}^{2}}{2}\right] \\
& =\Lambda .
\end{aligned}
$$

Then

$$
\begin{aligned}
& c_{1}=\frac{\Lambda}{\mathrm{d}+\sigma_{1}^{2} / 2+\sigma_{2}^{2} / 2}, \\
& c_{2}=\frac{\Lambda}{\left(\gamma+\delta+\mathrm{d}+\alpha_{1}\right)+\sigma_{1}^{2} / 2+\sigma_{3}^{2} / 2} .
\end{aligned}
$$


Thus we can get

$$
\begin{aligned}
L V_{1} & \leq-3\left[\left(\frac{\mathrm{d} \beta \Lambda^{3}}{\left(\mathrm{~d}+\sigma_{1}^{2} / 2+\sigma_{2}^{2} / 2\right)\left(\left(\gamma+\delta+\mathrm{d}+\alpha_{1}\right)+\sigma_{1}^{2} / 2+\sigma_{3}^{2} / 2\right)}\right)^{1 / 3}-\Lambda\right]+\frac{c_{1} \beta I}{S+I+R} \\
& \leq-3 \Lambda\left[\left(R_{0}^{s}\right)^{1 / 3}-1\right]+\frac{c_{1} \beta I}{S+I+R} .
\end{aligned}
$$

In addition, we can define

$$
\widehat{V}=M V_{1}-\ln S-\ln R-\ln Q+(S+I+Q+R) ;
$$

combined with (17), we can easily find that

$$
\lim _{s \rightarrow \infty,(S, I, Q, R) \in \mathbb{R}_{+}^{4} \backslash U_{s}} \widehat{V}(S, I, Q, R)=+\infty ;
$$

here $U_{s}=(1 / s, s) \times(1 / s, s) \times(1 / s, s) \times(1 / s, s)$. Moreover, $\widehat{V}(S, I, Q, R)$ is also a continuous function [25]. Therefore $\widehat{V}(S, I, Q, R)$ must have a minimum point $[25]\left(\bar{S}_{0}, \bar{I}_{0}, \bar{Q}_{0}, \bar{R}_{0}\right)$ which is inside $\mathbb{R}_{+}^{4}$. Then we define a nonnegative $C^{2}$-function $V: \mathbb{R}_{+}^{4} \rightarrow \mathbb{R}_{+}$as follows:

$$
V(S, I, Q, R)=\widehat{V}(S, I, Q, R)-\widehat{V}\left(\bar{S}_{0}, \bar{I}_{0}, \bar{Q}_{0}, \bar{R}_{0}\right) .
$$

Combined with the calculation method of stochastic differential, we get

$$
\begin{aligned}
& L V \leq M\left\{\left[-3 \Lambda\left(R_{0}^{s}\right)^{1 / 3}-1\right]+\frac{c_{1} \beta I}{S+I+R}\right\}-\frac{\Lambda}{S} \\
& +\frac{\beta I}{S+I+R}+\mathrm{d}+\frac{\sigma_{1}^{2}}{2}-\frac{\delta I}{\mathrm{Q}}+\left(\varepsilon+\mathrm{d}+\alpha_{2}\right) \\
& +\frac{\sigma_{3}^{2}}{2}-\frac{r I}{R}-\frac{\varepsilon Q}{R}+\mathrm{d}+\frac{\sigma_{4}^{2}}{2}+\Lambda \\
& -\mathrm{d}(S+I+Q+R)+\alpha_{1} I+\alpha_{2} Q \\
& \leq-M \lambda+\frac{M c_{1} \beta I}{S+I+R}+\frac{\beta I}{S+I+R}-\frac{\Lambda}{S}-\frac{\gamma I}{R}-\frac{\varepsilon Q}{R} \\
& -\frac{\delta I}{\mathrm{Q}}-\mathrm{d}(S+I+Q+R)-\alpha_{1} I-\alpha_{2} Q+\Lambda \\
& +3 \mathrm{~d}+\varepsilon+\beta+\alpha_{2}+\frac{\sigma_{1}^{2}+\sigma_{3}^{2}+\sigma_{4}^{2}}{2} \\
& :=-M \lambda+\frac{M c_{1} \beta I}{S+I+R}-\frac{\Lambda}{S}-\frac{\gamma I}{R}-\frac{\delta I}{Q} \\
& -\mathrm{d}(S+I+Q+R)+\Lambda+3 \mathrm{~d}+\varepsilon+\beta+\alpha_{2} \\
& +\frac{\sigma_{1}^{2}+\sigma_{3}^{2}+\sigma_{4}^{2}}{2} \text {. }
\end{aligned}
$$

Next we consider the following five cases. Let $K=M c_{1} \beta+$ $\Lambda+3 \mathrm{~d}+\mu+\beta+\alpha_{2}+\left(\sigma_{1}^{2}+\sigma_{2}^{2}+\sigma_{4}^{2}+\sigma_{5}^{2}\right) / 2$, where $K$ is positive constant.
Case 1 (if $\left.(S, I, Q, R) \in D_{1}\right)$. We get

$$
L V \leq K-\frac{\Lambda}{S} \leq K-\frac{\Lambda}{\varepsilon_{1}} ;
$$

combined with (13), we have

$$
L V \leq-1 \text { for any }(S, I, Q, R) \in D_{1} .
$$

Case 2 (if $\left.(S, I, Q, R) \in D_{2}\right)$. We can obtain

$$
\begin{aligned}
L V \leq & -M \lambda+\frac{M c_{1} \beta I}{S}+\Lambda+3 \mathrm{~d}+\mu+\beta+\alpha_{2} \\
& +\frac{\sigma_{1}^{2}+\sigma_{2}^{2}+\sigma_{4}^{2}+\sigma_{5}^{2}}{2} \\
\leq & -M \lambda+\frac{M c_{1} \beta \varepsilon_{1}^{2}}{\varepsilon_{1}}+\Lambda+3 \mathrm{~d}+\mu+\beta+\alpha_{2} \\
& +\frac{\sigma_{1}^{2}+\sigma_{2}^{2}+\sigma_{4}^{2}+\sigma_{5}^{2}}{2} .
\end{aligned}
$$

Thus one can see that

$$
\begin{aligned}
L V \leq & -M \lambda+M c_{1} \beta \varepsilon_{1}+\Lambda+3 \mathrm{~d}+\mu+\beta+\alpha_{2} \\
& +\frac{\sigma_{1}^{2}+\sigma_{2}^{2}+\sigma_{4}^{2}+\sigma_{5}^{2}}{2}
\end{aligned}
$$

combined with (12) and (17), for any sufficiently small $\varepsilon_{1}$, we get

$$
L V \leq-1 \text { for any }(S, I, Q, R) \in D_{2} \text {. }
$$

Case 3 (if $\left.(S, I, Q, R) \in D_{3}\right)$. We find that

$$
\begin{aligned}
L V \leq & -M \lambda+\frac{M c_{1} \beta I}{S+I+R}-\frac{\Lambda}{S}-\frac{\gamma I}{R}-\frac{\delta I}{Q} \\
& -\mathrm{d}(S+I+Q+R)+\Lambda+3 \mathrm{~d}+\mu+\beta+\alpha_{2} \\
& +\frac{\sigma_{1}^{2}+\sigma_{2}^{2}+\sigma_{4}^{2}+\sigma_{5}^{2}}{2} \leq K-\frac{\delta I}{Q} \leq K-\frac{\delta \varepsilon_{1}^{2}}{\varepsilon_{1}^{3}} \\
\leq & K-\frac{\delta}{\varepsilon_{1}} ;
\end{aligned}
$$

combined with (14), we have

$$
L V \leq-1 \quad \text { for any }(S, I, Q, R) \in D_{3} .
$$



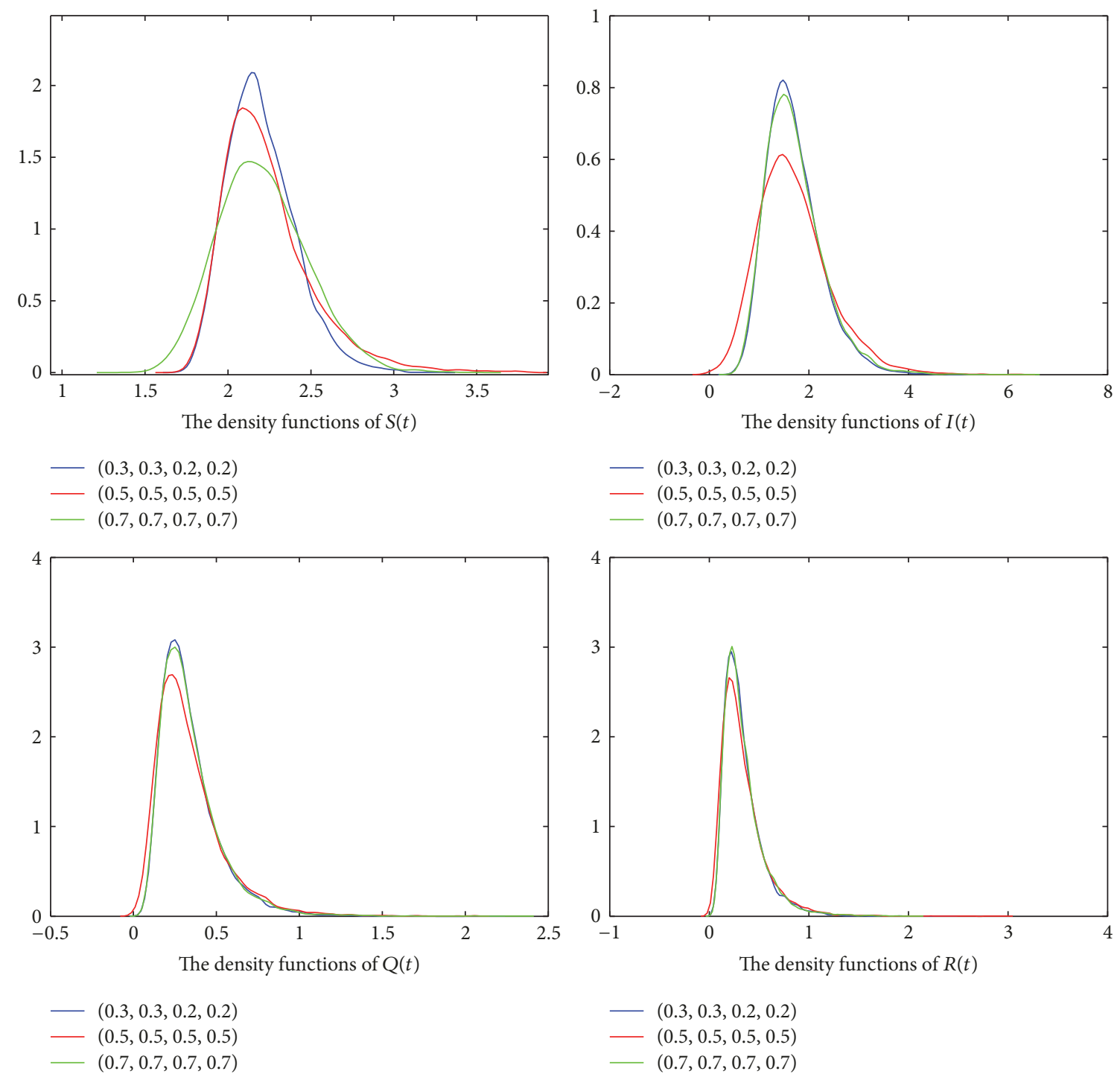

Figure 1: Based on the 10000 sample paths, after iterating 10,000 times, we get the density functions of $S(t), I(t), R(t)$ and $Q(t)$ with different initial values. Here $\Delta t=0.01$.

Case 4 (if $\left.(S, I, Q, R) \in D_{4}\right)$. One can see that

$$
\begin{aligned}
L V \leq & -M \lambda+\frac{M c_{1} \beta I}{S+I+R}-\frac{\Lambda}{S}-\frac{\gamma I}{R}-\frac{\delta I}{Q} \\
& -\mathrm{d}(S+I+Q+R)+\Lambda+3 \mathrm{~d}+\mu+\beta+\alpha_{2} \\
& +\frac{\sigma_{1}^{2}+\sigma_{2}^{2}+\sigma_{4}^{2}+\sigma_{5}^{2}}{2} \leq K-\frac{\gamma I}{R} \leq K-\frac{\gamma \varepsilon_{1}^{2}}{\varepsilon_{1}^{3}} \\
\leq & K-\frac{\gamma}{\varepsilon_{1}}
\end{aligned}
$$

combined with (15), we get

$$
L V \leq-1 \quad \text { for any }(S, I, Q, R) \in D_{4} \text {. }
$$

Case 5 (if $\left.(S, I, Q, R) \in D_{5}\right)$. We can obtain

$$
L V \leq K-\mathrm{d}(S+I+Q+R) \leq K-\frac{\mathrm{d}}{\varepsilon_{1}}
$$

according to (16), one can see that

$$
L V \leq-1 \quad \text { for any }(S, I, Q, R) \in D_{5} .
$$

Obviously, from (30), (33), (35), (37), and (39), we can easily find that for a sufficiently small $\varepsilon_{1}$,

$$
L V(S, I, Q, R) \leq-1 \quad \forall(S, I, Q, R) \in \mathbb{R}_{+}^{4} \backslash D .
$$

Thus we can easily find that $\left(A_{2}\right)$ in Lemma 1 is satisfied. From Lemma 1, we get that model (3) has a uniquely stationary distribution and satisfies ergodicity. Hence, the proof of Theorem 3 has been carried out. 

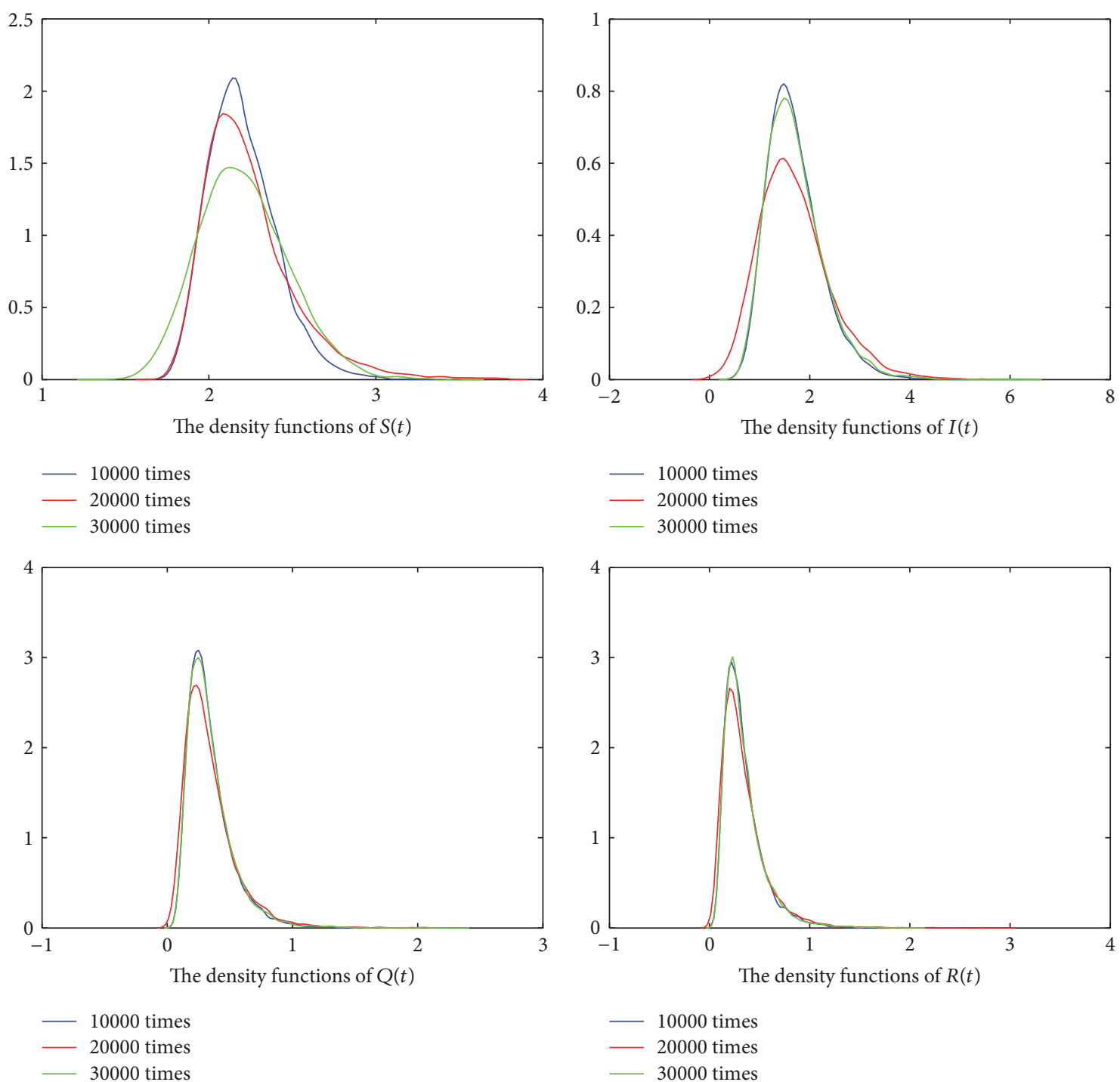

Figure 2: Based on the 10000 sample paths, after iterating 10,000 times, 20,000 times and 30,000 times, respectively, we get the density functions of $S(t), I(t), R(t)$ and $Q(t)$ with $S(0)=0.3, I(0)=0.3, Q(0)=0.2$, and $R(0)=0.2$. Here $\Delta t=0.01$.

\section{Disease Extinction}

In this section, we will investigate the extinction of disease under the following conditions:

$$
\begin{aligned}
& \left(C_{1}\right) \sigma_{1}^{2} \leq \beta \text { and } \widehat{R_{0}^{s}}<1, \text { where } \widehat{R_{0}^{s}}=\beta /\left(\gamma+\delta+\mathrm{d}+\alpha_{1}+\right. \\
& \left.\sigma_{1}^{2} / 2+\sigma_{3}^{2} / 2\right), \\
& \left(C_{2}\right) \sigma_{1}^{2}>\max \left\{\beta, \beta^{2} / 2\left(\gamma+\delta+\mathrm{d}+\alpha_{1}+\sigma_{3}^{2} / 2\right)\right\} .
\end{aligned}
$$

Theorem 4. If $\left(C_{1}\right)$ or $\left(C_{2}\right)$ holds, the disease $I(t)$ will die out exponentially with probability 1; that is,

$$
\lim _{t \rightarrow \infty} \sup \frac{\ln I(t)}{t}<0 \text { a.s. }
$$

Proof. By Itô’s formula, one can easily find that

$$
d \ln I(t)=\left[\frac{\beta S}{S+I+R}-\left(\gamma+\delta+\mathrm{d}+\alpha_{1}\right)\right.
$$

$$
\begin{aligned}
& \left.-\frac{\sigma_{1}^{2}}{2}\left(\frac{S}{S+I+R}\right)^{2}-\frac{\sigma_{3}^{2}}{2}\right] d t+\sigma_{1} \\
& \cdot \frac{S}{S+I+R} d B_{1}(t)+\sigma_{3} d B_{3}(t) .
\end{aligned}
$$

Integrating (43) from 0 to $t$, one can obtain that

$$
\begin{gathered}
\ln I(t)-\ln I(0)=\int_{0}^{t}\left[\frac{\beta S(u)}{S(u)+I(u)+R(u)}\right. \\
-\left(\gamma+\delta+\mathrm{d}+\alpha_{1}+\frac{\sigma_{3}^{2}}{2}\right) \\
\left.-\frac{\sigma_{1}^{2}}{2}\left(\frac{S(u)}{S(u)+I(u)+R(u)}\right)^{2}\right] d u+\int_{0}^{t} \sigma_{1} \\
\cdot \frac{S(u)}{S(u)+I(u)+R(u)} d B_{1}(u)+\sigma_{3} B_{3}(u) .
\end{gathered}
$$





FIGURE 3: The blue line corresponds to the density function of the solution with $S(0)=0.3, I(0)=0.3, Q(0)=0.2$, and $R(0)=0.2$. Based on the 10000 sample paths, after iterating 10,000 times. The red line corresponds to the density function of all states that one trajectory of model (3) reaches. Here $\Delta t=1$.

Let

$$
\begin{aligned}
& x=\frac{S(u)}{S(u)+I(u)+R(u)}, \quad x \in(0,1], \\
& C=\gamma+\delta+\mathrm{d}+\alpha_{1}+\frac{\sigma_{3}^{2}}{2} .
\end{aligned}
$$

We can obtain

$$
\begin{aligned}
& \frac{\beta S(u)}{S(u)+I(u)+R(u)}-\left(\gamma+\delta+\mathrm{d}+\alpha_{1}+\frac{\sigma_{3}^{2}}{2}\right) \\
& -\frac{\sigma_{1}^{2}}{2}\left(\frac{S(u)}{S(u)+I(u)+R(u)}\right)^{2}=\beta x-C+\frac{\sigma_{1}^{2}}{2} x^{2} \\
& \quad=f(x), \\
& f^{\prime}(x)=\beta-\sigma^{2} x .
\end{aligned}
$$

Next, let $f^{\prime}(x)=0$, then one can get $x=\beta / \sigma_{1}^{2}$.

Case 1. Suppose that $\left(C_{1}\right)$ holds; we can get

$$
f(x) \leq f(1)=\beta-\mathrm{C}+\frac{\sigma_{1}^{2}}{2}
$$

thus we have

$$
\begin{aligned}
\ln I( & t)-\ln I(0) \\
\leq & \int_{0}^{t}\left[\beta-\left(\gamma+\delta+\mathrm{d}+\alpha_{1}+\frac{\sigma_{3}^{2}}{2}+\frac{\sigma_{1}^{2}}{2}\right)\right] d u \\
& +\sigma_{1} B_{1}(t)+\sigma_{3} B_{3}(t) \\
= & \left(\gamma+\delta+\mathrm{d}+\alpha_{1}+\frac{\sigma_{3}^{2}}{2}+\frac{\sigma_{1}^{2}}{2}\right)\left(\widehat{R_{0}^{s}}-1\right) t \\
& \quad+\sigma_{1} B_{1}(t)+\sigma_{3} B_{3}(t) .
\end{aligned}
$$

By the strong law of large numbers (see [8]), we get that

$$
\lim _{t \rightarrow \infty} \frac{B_{i}(t)}{t}=0 \quad i=1,3 \text { a.s. }
$$

Both sides simultaneously take maximum of (49) and combining with (50), we have

$$
\begin{aligned}
& \underset{t \rightarrow \infty}{\lim \sup } \frac{\ln I(t)}{t} \\
& \quad \leq\left(\gamma+\delta+\mathrm{d}+\alpha_{1}+\frac{\sigma_{3}^{2}}{2}+\frac{\sigma_{1}^{2}}{2}\right)\left(\widehat{R_{0}^{s}}-1\right)<0 \text { a.s.; }
\end{aligned}
$$

this means that $\lim _{t \rightarrow \infty} I(t)=0$ a.s.. In other words, the disease $I(\mathrm{t})$ will tend to zero exponentially with probability 1 . 




$-S(t)$

(a) Beta $=0.4$

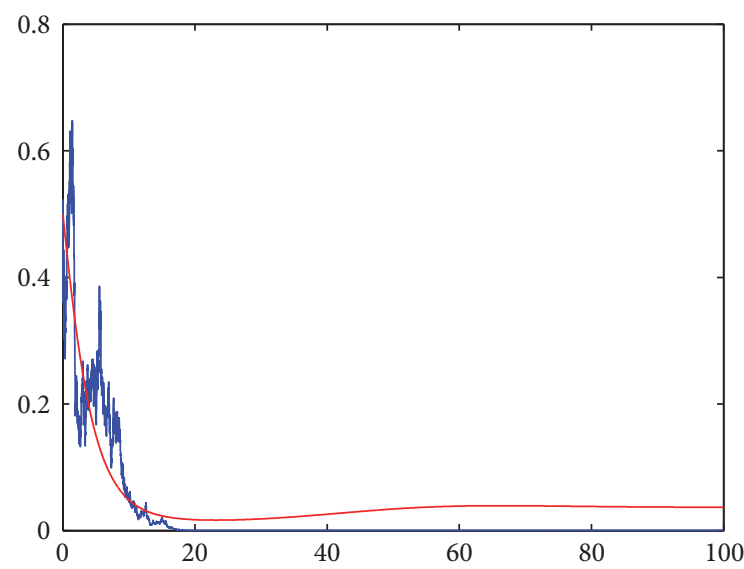

- $Q(t)$

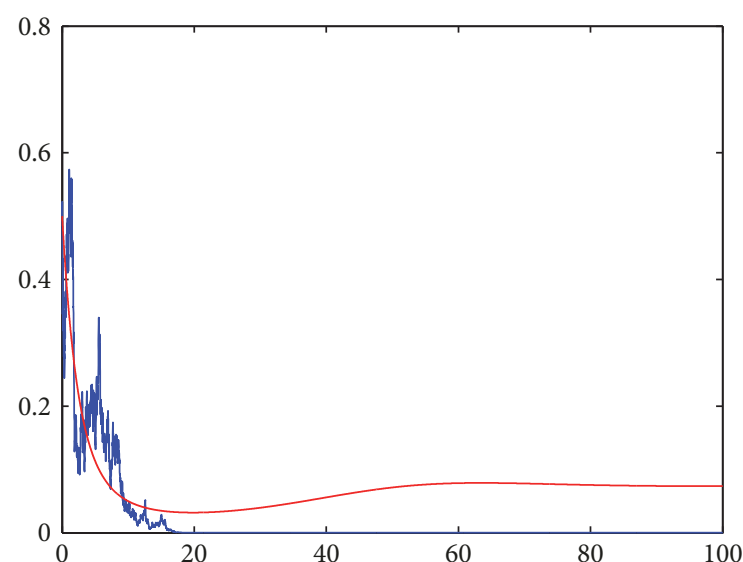

$I(t)$

(b) Beta $=0.4$



$R(t)$

(c) Beta $=0.4$

(d) Beta $=0.4$

FIGURE 4: Computer simulation of the paths $S(t), I(t), Q(t)$, and $R(t)$ for models (1) and (3) for parameter values $\Lambda=0.2 ; d=0.1 ; \beta=$ $0.7 ; \gamma=0.2 ; \delta=0.2 ; \alpha_{1}=0.1 ; \sigma_{1}=0.4 ; \sigma_{2}=0.1 ; \sigma_{3}=0.4 ; \mu=0.1$; this way, $\sigma_{1}^{2} \leq \beta$ and $\widehat{R_{0}^{s}}<1$ hold.

Case 2. If $\left(C_{2}\right)$ holds, this conclusion is also correct. In fact, by the condition, we can get

$$
f(x) \leq f\left(\frac{\beta}{\sigma_{1}^{2}}\right)=\frac{\beta^{2}}{\sigma_{1}^{2}}-C+\frac{\sigma_{1}^{2}}{2}\left(\frac{\beta}{\sigma_{1}^{2}}\right)^{2}=\frac{\beta^{2}}{2 \sigma_{1}^{2}}-C ;
$$

thus we have

$$
\begin{aligned}
& \ln I(t)-\ln I(0) \\
& \leq \int_{0}^{t}\left[\frac{\beta^{2}}{2 \sigma_{1}^{2}}-\left(\gamma+\delta+\mathrm{d}+\alpha_{1}+\frac{\sigma_{3}^{2}}{2}\right)\right] d u \\
& \quad+\sigma_{1} B_{1}(t)+\sigma_{3} B_{3}(t) \\
& =\left[\frac{\beta^{2}}{2 \sigma_{1}^{2}}-\left(\gamma+\delta+\mathrm{d}+\alpha_{1}+\frac{\sigma_{3}^{2}}{2}\right)\right] t+\sigma_{1} B_{1}(t) \\
& \quad+\sigma_{3} B_{3}(t) .
\end{aligned}
$$

Both sides simultaneously take maximum of (53) and combining with (50), we get

$$
\begin{aligned}
\limsup _{t \rightarrow \infty} \frac{\ln I(t)}{t} & \leq\left[\frac{\beta^{2}}{2 \sigma_{1}^{2}}-\left(\gamma+\delta+\mathrm{d}+\alpha_{1}+\frac{\sigma_{3}^{2}}{2}\right)\right] \\
& <0 \text { a.s. }
\end{aligned}
$$

By system (3), one can easily obtain that when $\lim _{t \rightarrow \infty} I(t)=0$ a.s., then $\lim _{t \rightarrow \infty} Q(t)=0$ and $\lim _{t \rightarrow \infty} R(t)=0$ a.s.. This completes the proof.

Remark 5. From condition $\left(C_{1}\right)$, we can easily find that if $\widehat{R_{0}^{s}}<1$ and white noise is small, the disease will be extinct. From condition $\left(C_{2}\right)$ we get that if white noise is large enough, the disease will also be extinct. Otherwise, the manifestation of the disease is uncertain, which does not happen in deterministic system (1). Furthermore, we notice that $\widehat{R_{0}^{s}}$ in $C_{1}$ is smaller than the basic reproduction number of system (1). 


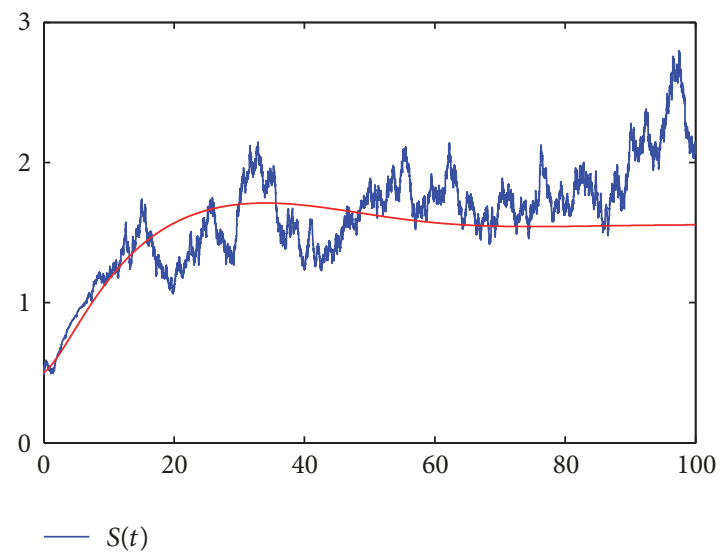

(a) Beta $=0.4$

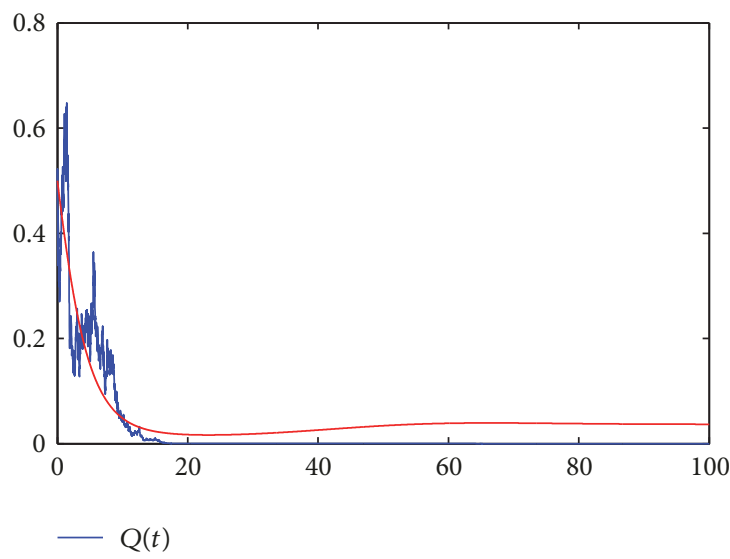

(c) Beta $=0.4$

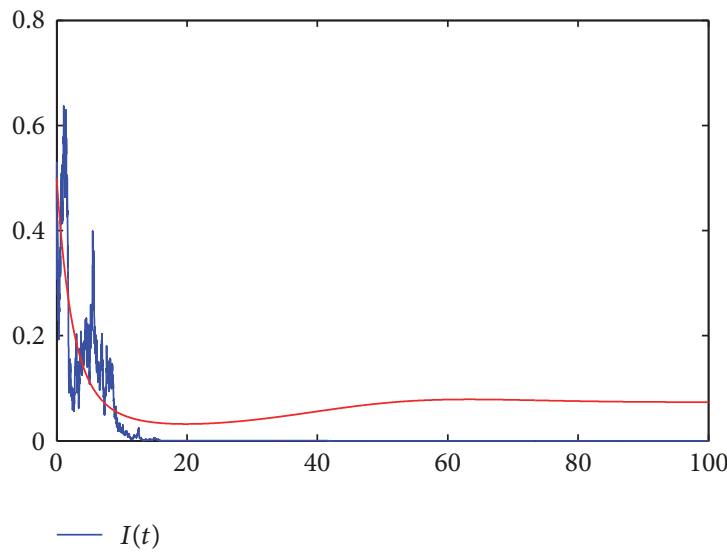

(b) Beta $=0.4$

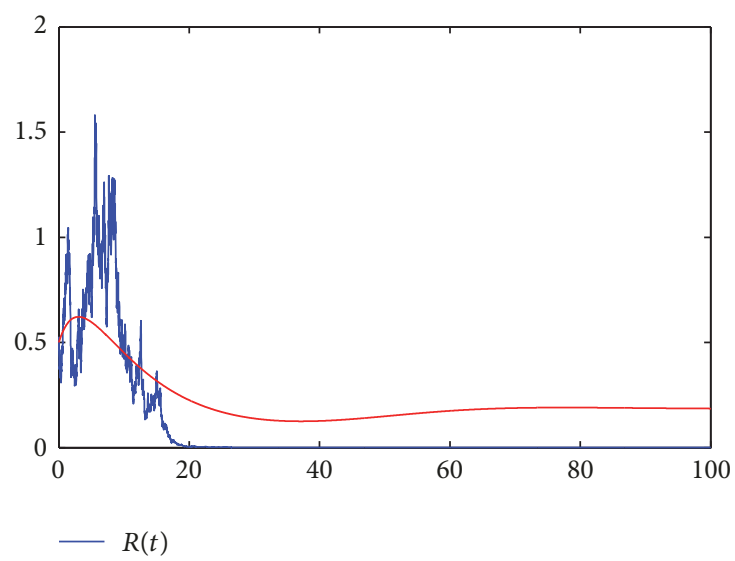

(d) Beta $=0.4$

FIGURE 5: Computer simulation of the paths $S(t), I(t), Q(t)$, and $R(t)$ for models (1) and (3) for parameter values $\Lambda=0.2 ; d=0.1 ; \beta=$ $0.7 ; \gamma=0.2 ; \delta=0.2 ; \alpha_{1}=0.1 ; \sigma_{1}=0.85 ; \sigma_{2}=0.1 ; \sigma_{3}=0.4 ; \mu=0.1$, such that $\sigma_{1}^{2}>\max \left\{\beta, \beta^{2} / 2\left(\gamma+\delta+\mathrm{d}+\alpha_{1}+\sigma_{3}^{2} / 2\right)\right\}$ hold.

\section{Examples and Numerical Simulations}

In this section, we will give some numerical examples to illustrate our main results by using Milstein's Higher Order Method [28].

Example 1. We choose the parameter values in system (3) as follows: $\Lambda=1 ; d=0.2 ; \beta=0.66 ; \gamma=0.02 ; \delta=0.1 ; \alpha_{1}=$ $0.01 ; \mu=0.1 ; \alpha_{2}=0.2 ; \sigma_{1}=0.01 ; \sigma_{2}=0.002 ; \sigma_{3}=$ $0.2 ; \sigma_{4}=0.3 ; \sigma_{5}=0.2$. By calculation, we can get $R_{0}^{s}=$ $1.8850>1$. That is to say, the conditions of Theorem 3 are satisfied. In Figure 1, we choose the different initial values to illustrate that wherever $S(t), I(t), R(t)$ and $Q(t)$ start from, the density functions of $S(t), I(t), R(t)$ and $Q(t)$ converge to the same functions, respectively. In Figure 2, selecting different iterations, one can easily find that the density functions of $S(t), I(t), R(t)$, and $Q(t)$ also converge to the same functions, respectively. Hence, Figures 1 and 2 verify Theorem 3 very well so that there exists a unique ergodic stationary distribution $\mu(\cdot)$ of system (3). In Figure 3, the blue line and red line are almost the same. This strongly illustrates ergodicity.
Example 2. In order to obtain the extinction of the model, we give numerical simulations under which one of the conditions of $C_{1}$ and $C_{2}$ hold. In addition, neither $C_{1}$ nor $C_{2}$ holds; we also give numerical simulations to verify that in this case, the disease may be persistent in Figure 6 or extinct in Figure 7.

In Figure 4 , the parameters in (3) are chosen by $\Lambda=$ $0.2 ; d=0.1 ; \beta=0.7 ; \gamma=0.2 ; \delta=0.2 ; \alpha_{1}=0.1 ; \mu=$ $0.1 ; \alpha_{2}=0.2 ; \sigma_{1}=0.4 ; \sigma_{2}=0.1 ; \sigma_{3}=0.4 ; \sigma_{4}=0.5 ; \sigma_{5}=$ 0.5 . By calculation, we know that $\sigma_{1}{ }^{2}=0.16<\beta$ and $\widehat{R_{0}^{s}}=$ $0.9211<1$. This means that $C_{1}$ holds. In Figure 5, we keep all the parameters unchanged but increase $\sigma_{1}$ to 0.85 . Note that $\beta^{2} / 2\left(\gamma+\delta+d+\alpha_{1}+\sigma_{3}^{2} / 2\right)=0.3603$ and $\sigma_{1}{ }^{2}=0.7225>\beta>$ 0.3603 , which means that $C_{2}$ holds in Theorem 4 . Figures 4 and 5 verify Theorem 4 very well so that if $\left(C_{1}\right)$ or $\left(C_{2}\right)$ holds, $I(t)$ will tend to zero exponentially with probability one.

In Figure 6, by changing $\gamma=0.1, \delta=0.2$, and $\sigma_{1}=0.1$ and not changing other parameters in Figure 4 , we calculate $\beta^{2} / 2\left(\gamma+\delta+d+\alpha_{1}+\sigma_{3}^{2} / 2\right)=0.5833$ and $0.01=\sigma_{1}^{2}<0.5833<$ $\beta=0.7$ and $\widehat{R_{0}^{s}}=1.6471>1$, which means that both $\left(C_{1}\right)$ and 




(a) Beta $=0.4$

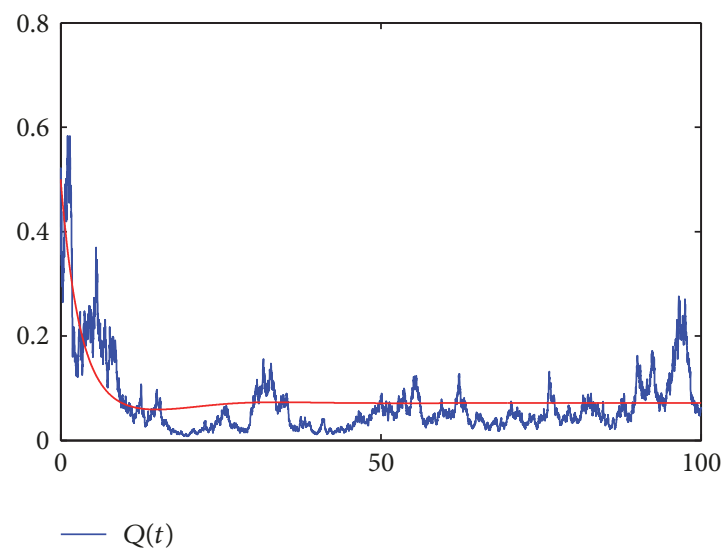

(c) Beta $=0.4$

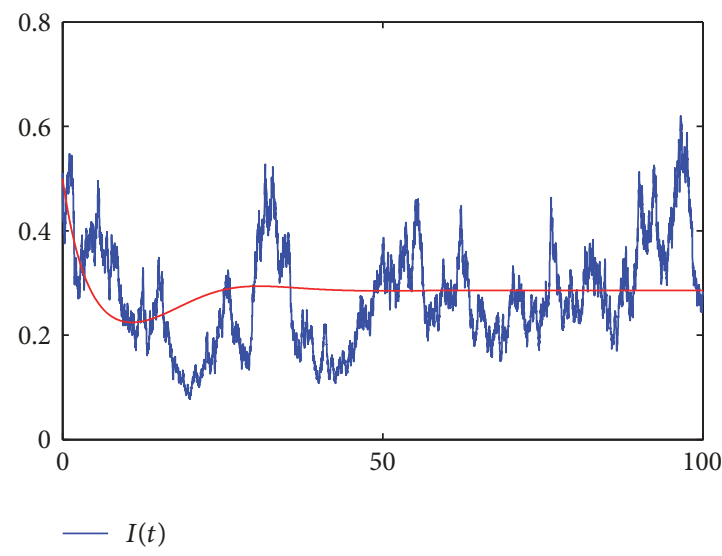

(b) Beta $=0.4$

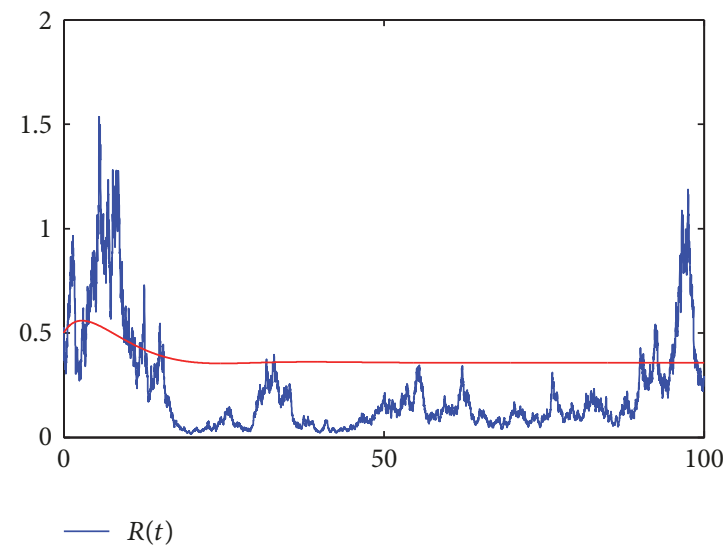

(d) Beta $=0.4$

FIGURE 6: Computer simulation of the paths $S(t), I(t), Q(t)$, and $R(t)$ for models (1) and (3) for parameter values $\Lambda=0.2 ; d=0.1 ; \beta=$ $0.7 ; \gamma=0.1 ; \delta=0.1 ; \alpha_{1}=0.1 ; \sigma_{1}=0.1 ; \sigma_{2}=0.1 ; \sigma_{3}=0.2 ; \mu=0.1$, such that $\sigma_{1}^{2}<\max \left\{\beta, \beta^{2} / 2\left(\gamma+\delta+\mathrm{d}+\alpha_{1}+\sigma_{3}^{2} / 2\right)\right\}$ and $\widehat{R_{0}^{s}}>1$ hold.

$\left(C_{2}\right)$ in Theorem 4 do not hold. From the line in Figure 6, we can easily find that $I(t)$ persist. In Figure 7, we also change some parameters $\gamma=0.1, \alpha_{1}=0.2, \sigma_{1}=0.2, \sigma_{3}=0.2$ and other parameters are unchanged in Figure 4. By calculation, we have $\sigma_{1}^{2}<\max \left\{\beta, \beta^{2} / 2\left(\gamma+\delta+d+\alpha_{1}+\sigma_{3}^{2} / 2\right)\right\}$ and $\widehat{R_{0}^{s}}=1.0937>1$, which also means that both $\left(C_{1}\right)$ and $\left(C_{2}\right)$ in Theorem 4 do not hold. From Figure 7, it is shown that $I(t)$ become extinct. Thus, combining Figure 6 with Figure 7, one can easily find that both $\left(C_{1}\right)$ and $\left(C_{2}\right)$ do not hold; the direction of $I(t)$ is uncertain and may persist or become extinct.

\section{Conclusion Remarks and Future Directions}

In this work, we study the dynamics of a stochastic SIQR epidemic disease with quarantine-adjusted incidence. After formulating the Lyapunov functions, we have confirmed existence and uniqueness of solutions of stochastic SIQR systems and basic reproduction number $R_{0}^{s}$. If $R_{0}^{s}>1$, there exists a stationary distribution $\pi(\cdot)$ and the ergodicity holds for any initial value in system (3). In addition, in the fourth part, when condition $\left(C_{1}\right)$ or $\left(C_{2}\right)$ holds, the disease $I(t)$ will be extinct.
In the future, we can propose a class of practical and complex models, such as considering the effects of regime switching on SIQR epidemic model (see [29]) or considering the dynamical properties of stochastic SIQR epidemic model with different incidence and time delays (see $[30,31]$ ) or the persistence and the existence of periodic solutions of stochastic $S I Q R$ epidemic model. We leave these investigations for future work.

\section{Conflicts of Interest}

The authors declare that they have no conflicts of interest.

\section{Acknowledgments}

This work was supported by National Natural Science Foundation of China (no. 11701209), Project of Science and Technology Development Plan for Jilin Province (no. 20160520110JH), the Key Project of Jilin University of Finance and Economics (no. 0800091602), and Science and Technology Research Project of Jilin Provincial Department of Education of China (research on qualitative property and numerical optimization methods of singular nonlinear differential equation). 


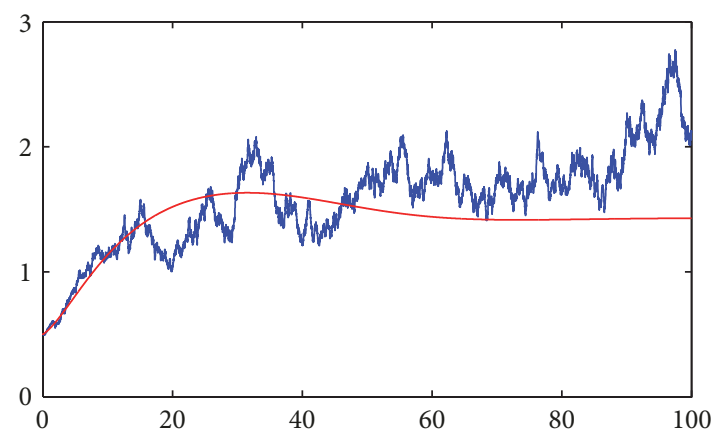
$-S(t)$

(a) Beta $=0.4$



(c) Beta $=0.4$
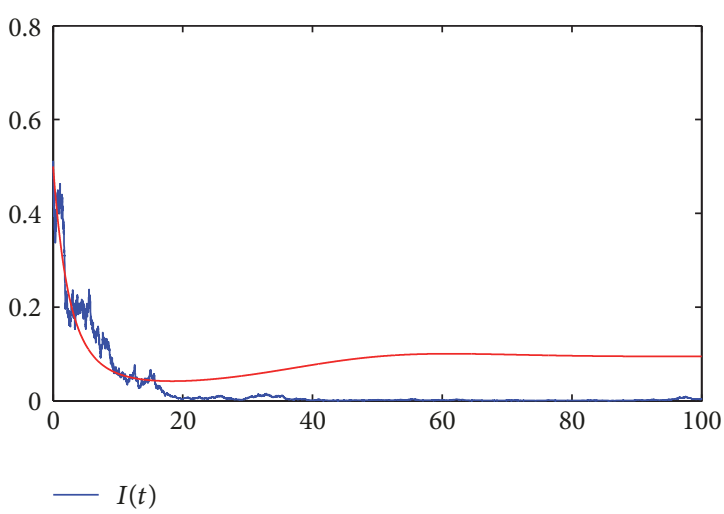

(b) Beta $=0.4$

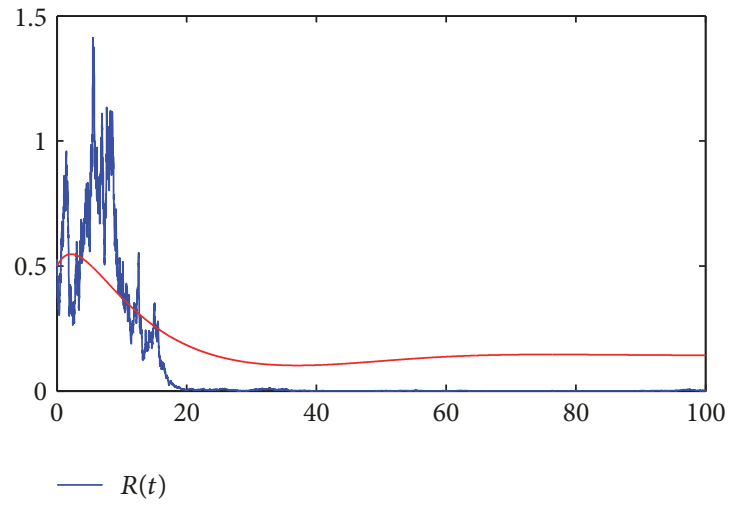

(d) Beta $=0.4$

Figure 7: Computer simulation of the paths $S(t), I(t), Q(t)$, and $R(t)$ for models (1) and (3) for parameter values $\Lambda=0.2 ; d=0.1 ; \beta=$ $0.7 ; \gamma=0.1 ; \delta=0.2 ; \alpha_{1}=0.2 ; \sigma_{1}=0.2 ; \sigma_{2}=0.1 ; \sigma_{3}=0.2 ; \mu=0.1$, such that $\sigma_{1}^{2}<\max \left\{\beta, \beta^{2} / 2\left(\gamma+\delta+\mathrm{d}+\alpha_{1}+\sigma_{3}^{2} / 2\right)\right\}$ and $\widehat{R_{0}^{s}}>1$ hold.

\section{References}

[1] X.-B. Zhang, H.-F. Huo, H. Xiang, and X.-Y. Meng, "Dynamics of the deterministic and stochastic SIQS epidemic model with non-linear incidence," Applied Mathematics and Computation, vol. 243, pp. 546-558, 2014.

[2] F. Wei and F. Chen, "Stochastic permanence of an SIQS epidemic model with saturated incidence and independent random perturbations," Physica A: Statistical Mechanics and its Applications, vol. 453, pp. 99-107, 2016.

[3] Q. Liu, D. Jiang, N. Shi, T. Hayat, and A. Alsaedi, "Stationary distribution and extinction of a stochastic SIRS epidemic model with standard incidence," Physica A: Statistical Mechanics and its Applications, vol. 469, pp. 510-517, 2017.

[4] A. S. Ackleh and L. J. Allen, "Competitive exclusion and coexistence for pathogens in an epidemic model with variable population size," Journal of Mathematical Biology, vol. 47, no. 2, pp. 153-168, 2003.

[5] H. A. A. El-Saka, "The fractional-order SIR and SIRS epidemic models with variable population," Applied Mathematics Letters, vol. 2, no. 3, pp. 195-200, 2013.

[6] W. O. Kermack and A. G. McKendrick, "Contributions to the mathematical theory of epidemics-I," Bulletin of Mathematical Biology, vol. 53, no. 1-2, pp. 33-55, 1991.

[7] Z. Ma, Y. Zhou, and J. Wu, Modeling and dynamics of infectious diseases, Higher Education Press, 2009.
[8] J. Zhang, Z. Jin, G.-Q. Sun, T. Zhou, and S. Ruan, "Analysis of rabies in China: transmission dynamics and control," PLoS ONE, vol. 6, no. 7, p. e20891, 2011.

[9] H. Hethcote, M. Zhien, and L. Shengbing, "Effects of quarantine in six endemic models for infectious diseases," Mathematical Biosciences, vol. 180, pp. 141-160, 2002.

[10] L. J. Allen, "An introduction to stochastic epidemic models," in Mathematical Epidemiology, vol. 2008 of Lecture Notes in Math., pp. 81-130, Springer, 1945.

[11] H. C. Tuckwell and R. J. Williams, "Some properties of a simple stochastic epidemic model of SIR type," Mathematical Biosciences, vol. 208, no. 1, pp. 76-97, 2007.

[12] R. Z. Hasminskii, "Stochastic Stability of Differential Equations," Sijthoff and Noordhoff, 1980.

[13] X. Mao, Stochastic Differential Equations and Their Applications, Horwood, Chichester, UK, 1997.

[14] X. Mao, G. Marion, and E. Renshaw, "Environmental Brownian noise suppresses explosions in population dynamics," Stochastic Processes and Their Applications, vol. 97, no. 1, pp. 95-110, 2002.

[15] Q. Yang, D. Jiang, N. Shi, and C. Ji, “The ergodicity and extinction of stochastically perturbed SIR and SEIR epidemic models with saturated incidenc," Journal of Mathematical Analysis and Applications, vol. 388, no. 1, pp. 248-271, 2012.

[16] R. Z. Khasminskii and F. C. Klebaner, "Long term behavior of solutions of the Lotka-Volterra system under small random perturbations," The Annals of Applied Probability, vol. 11, no. 3, pp. 952-963, 2001. 
[17] D. Li, J. Cui, M. Liu, and S. Liu, "The evolutionary dynamics of stochastic epidemic model with nonlinear incidence rate," Bulletin of Mathematical Biology, vol. 77, no. 9, pp. 1705-1743, 2015.

[18] Q. Yang and X. Mao, "Extinction and recurrence of multigroup SEIR epidemic models with stochastic perturbations," Nonlinear Analysis: Real World Applications, vol. 14, no. 3, pp. 1434-1456, 2013.

[19] T. Britton and D. Lindenstrand, "Epidemic modelling: aspects where stochasticity matters," Mathematical Biosciences, vol. 222, no. 2, pp. 109-116, 2009.

[20] J. A. Jacquez and P. O'Neill, "Reproduction numbers and thresholds in stochastic epidemic models I. Homogeneous populations," Mathematical Biosciences, vol. 107, no. 2, pp. 161186, 1991.

[21] O. A. van Herwaarden and J. Grasman, "Stochastic epidemics: major outbreaks and the duration of the endemic period," Journal of Mathematical Biology, vol. 33, no. 6, pp. 581-601, 1995.

[22] D. Jiang, C. Ji, N. Shi, and J. Yu, "The long time behavior of DI SIR epidemic model with stochastic perturbation," Journal of Mathematical Analysis and Applications, vol. 372, no. 1, pp. 162-180, 2010.

[23] Z. Liu, "Dynamics of positive solutions to SIR and SEIR epidemic models with saturated incidence rates," Nonlinear Analysis: Real World Applications, vol. 14, no. 3, pp. 1286-1299, 2013.

[24] C. Ji and D. Jiang, "Threshold behaviour of a stochastic SIR model," Applied Mathematical Modelling, vol. 38, no. 21-22, pp. 5067-5079, 2014.

[25] Q. Han, L. Chen, and D. Jiang, "A note on the stationary distribution of stochastic SEIR epidemic model with saturated incidence rate," Scientific Reports, vol. 7, no. 1, article no. 3996, 2017.

[26] K. Ito and S. Watanabe, Introduction to Stochastic Differential Equations, Marcel Dekker, New York, NY, USA, 1988.

[27] M.-C. Casabán, J.-C. Cortés, J.-V. Romero, and Roselló, “Probabilistic solution of random SI-type epidemiological models using the random variable transformation technique," Communications in Nonlinear Science and Numerical Simulation, vol. 24, no. 1-3, pp. 86-97, 2015.

[28] D. J. Higham, "An algorithmic introduction to numerical simulation of stochastic differential equations," SIAM Review, vol. 43, no. 3, pp. 525-546, 2001.

[29] Q. Liu, D. Jiang, and N. Shi, “Threshold behavior in a stochastic SIQR epidemic model with standard incidence and regime switching," Applied Mathematics and Computation, vol. 316, pp. 310-325, 2018.

[30] M. Liu and M. Fan, "Stability in distribution of a three-species stochastic cascade predator-prey system with time delays," IMA Journal of Applied Mathematics, vol. 82, no. 2, pp. 396-423, 2017.

[31] M. Liu, C. Bai, and Y. Jin, "Population dynamical behavior of a two-predator one-prey stochastic model with time delay," Discrete and Continuous Dynamical Systems - Series A, vol. 37, no. 5, pp. 2513-2538, 2017. 


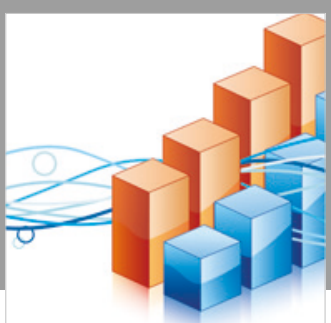

Advances in

Operations Research

\section{-n-m}
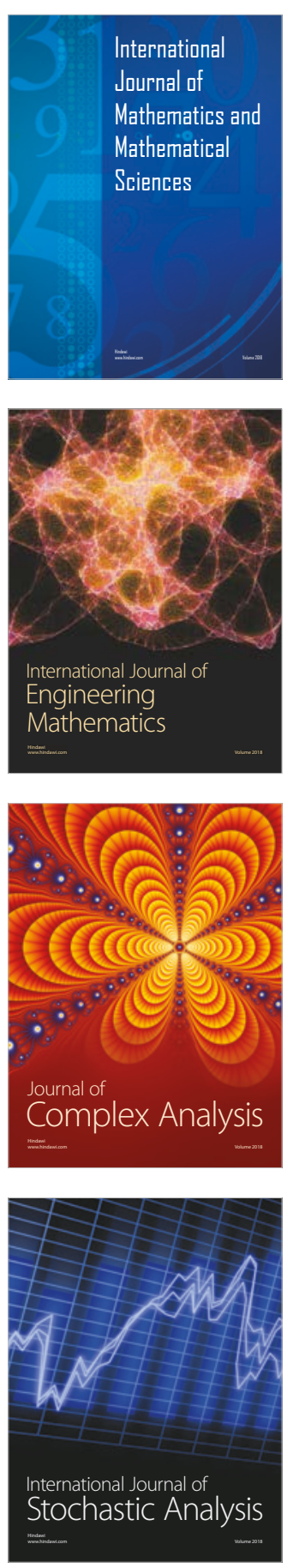
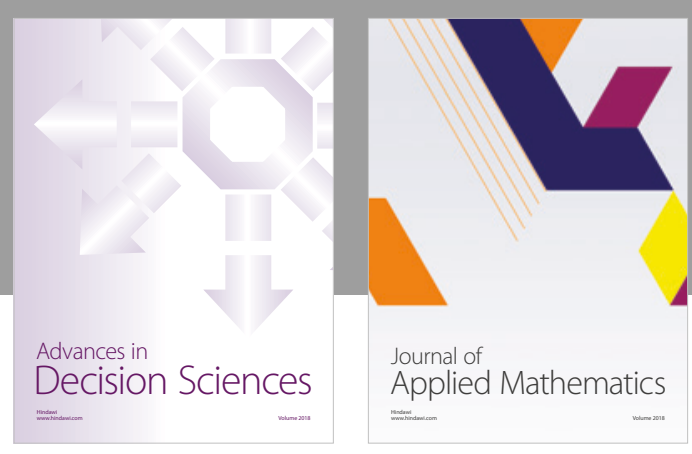

Journal of

Applied Mathematics
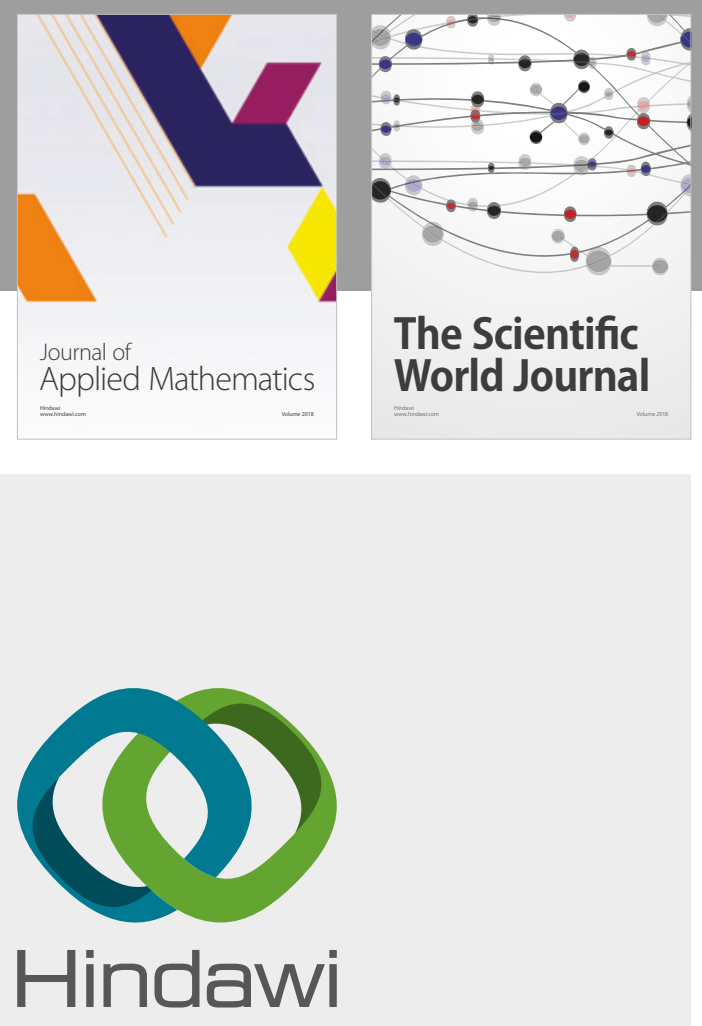

Submit your manuscripts at

www.hindawi.com

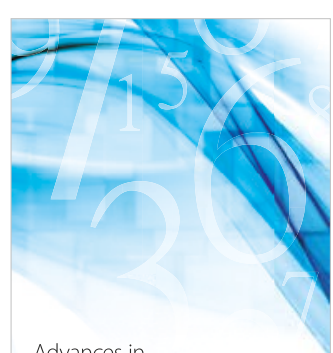

Advances in
Numerical Analysis
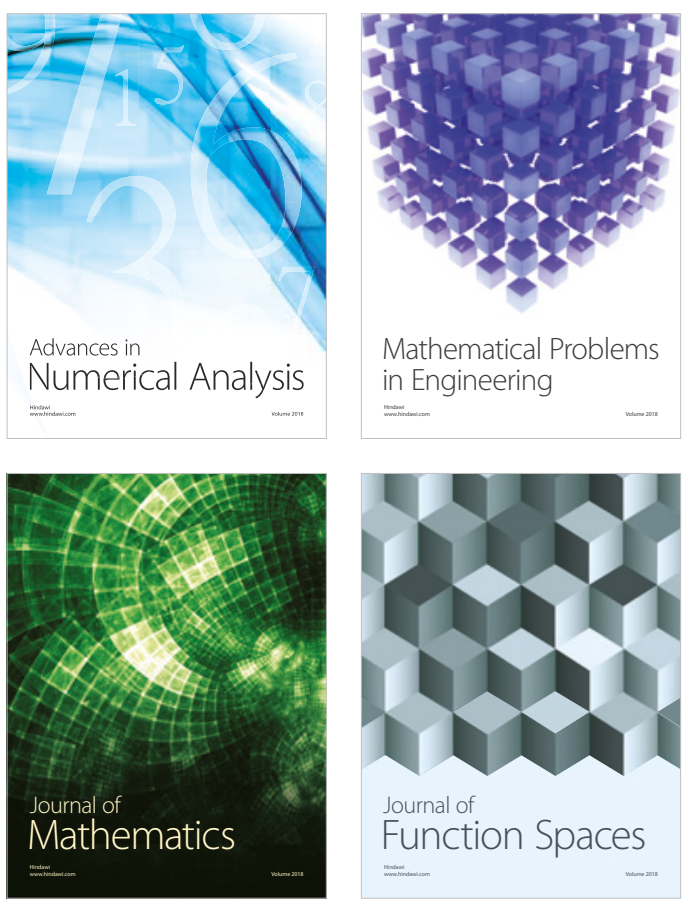

Mathematical Problems in Engineering



International Journal of

Differential Equations

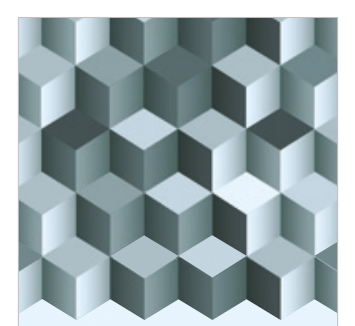

Journal of

Function Spaces

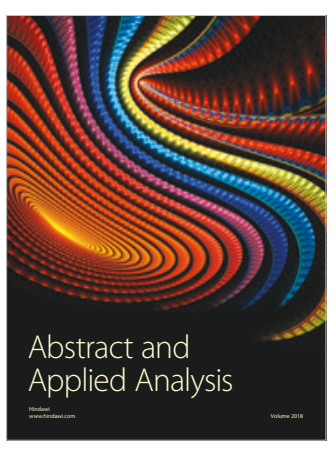

The Scientific

World Journal

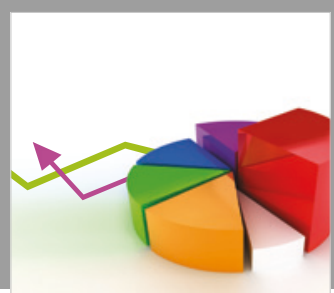

Journal of

Probability and Statistics
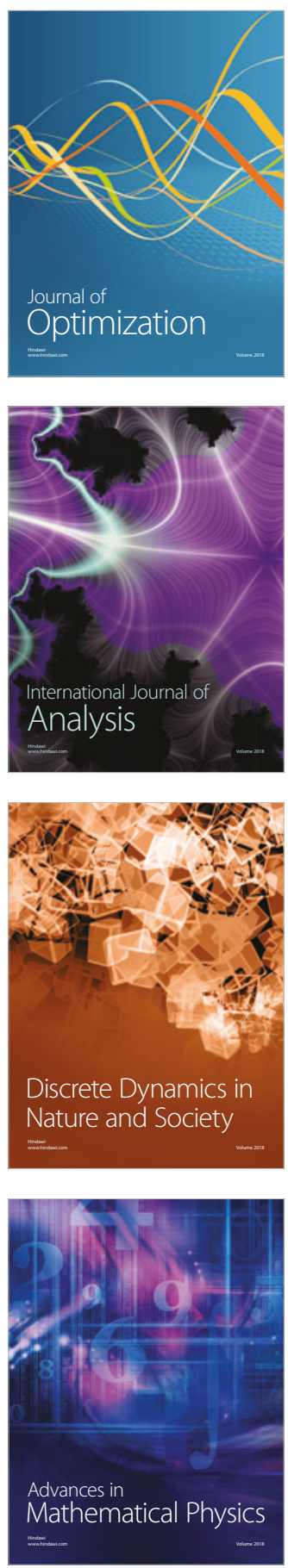